\title{
DEMANDAS EN EL CIADI Y EL DERECHO HUMANO AL AGUA: ¿TRATADOS DE INVERSIONES VS. DERECHOS HUMANOS?*
}

\author{
CASES AT ICSID AND THE HUMAN \\ RIGHT TO WATER: INVESTMENT \\ TREATIES VS. HUMAN RIGHTS?
}

JAVIER ECHAIDE**

Fecha de recepción: 24 de septiembre de 2016

Fecha de aceptación: 1 de diciembre de 2016

Disponibilidad en línea: 30 de diciembre de 2016

\section{Para citar este artículo / To cite this article}

Echaide, Javier, Demandas en el CIADI y el derecho humano al agua: ¿tratados de inversión vs. derechos humanos?, 31 International Law, Revista Colombiana de Derecho Internacional, 81-114(2016). https://doi.org/10.11144/ Javeriana.il 14-31.dcdh

doi:10.11144/Javeriana.il14-31.dcdh

* Investigación, adscripta a la Universidad Argentina de la Empresa, UADE.

** Abogado, Universidad de Buenos Aires, UBA. Doctor en derecho, Universidad de Buenos Aires, UBA. Actualmente, es profesor de ciencias jurídicas e investigador del Instituto de Ciencias Sociales y Disciplinas Proyectuales, INSOD, en la Universidad Argentina de la Empresa, UADE, Buenos Aires, Argentina. Contacto: jaechaide@uade.edu.ar 


\section{RESUMEN}

Argentina es el país más demandado ante el Centro Internacional de Arreglo de Diferencias sobre Inversiones (CIADI) y uno de los que más Tratados Bilaterales de Inversión (TBI) ha firmado (58 en total, 55 vigentes). La mayoría de esas demandas fueron presentadas por empresas transnacionales (ETN) por la crisis de 2001-2002 y se refirieron a sectores económicos relacionados con recursos naturales. E1 20\% de esas demandas se vincula con el sector de agua potable y saneamiento, lo que también convierte a Argentina en el país más demandado en el CIADI por este sector. Son casos de alta conflictividad social y en los que se evidencia el rol del Estado como regulador frente al reclamo por parte de las ETN de protección de la propiedad privada, en los que resalta una asimetría jurídica que favorece la protección de las inversiones sobre el resguardo de los derechos humanos, en particular del derecho humano al agua reconocido por la Organización de Naciones Unidas, ONU, en 2010. Tomando el caso argentino como testigo, esta ponencia expondrá esa asimetría que contraviene las bases más elementales del derecho internacional, para evidenciar la necesidad de revisar el régimen internacional de protección de inversiones y su adecuación a los derechos humanos como obligaciones erga omnes.

Palabras clave: Inversiones extranjeras; tratados; empresas transnacionales; derechos humanos; mercados del agua 


\section{ABSTRACT}

Argentina is the most sued country in the International Centre for Settlement of Investment Disputes (ICSID) and one of the most Bilateral Investment Treaties (BITs) signed (58 in total, 55 into force). Most of these lawsuits were filed by transnational corporations (TNCs) by the 2001-2002 crisis and linked to economic sectors related to natural resources. $20 \%$ of these claims are related to the drinking water and sanitation, which also makes Argentina in the country that has received claims in ICSID this sector. These are cases of social conflict and where the role of the state is evident as a regulator against the claim of protection of private property by TNCs, which highlights a legal asymmetry that favors the protection of investments on the protection of rights humans, particularly the human right to water recognized by the UN in 2010. Taking the Argentine case as a witness, this paper will present an asymmetry that violates the most basic foundations of international law, highlighting the need to revise the international investment protection regime and its relevance to the human rights obligations erga omnes.

Keywords: International investments; Treaties; Transnational corporations; Human Rights; Water markets

\section{SUMARIO}

INTRODUCCIÓN.- I. LA RELACIÓN ENTRE AGUA, LOS DERECHOS HUMANOS Y LAS INVERSIONES.- II. INFLACIÓN Y ASIMETRÍA NORMATIVA EN EL DERECHO INTERNACIONAL.- III. LA FRAGMENTACIÓN DEL DERECHO INTERNACIONAL.- IV. UN DESARROLLO PROGRESIVO DE MÚLTIPLES VELOCIDADES.- CONCLUSIONES.- BIBLIOGRAFÍA. 
INTRODUCCIÓN ${ }^{1}$

El derecho internacional presenta un problema que viene acentuándose cada vez más a medida que este se desarrolla. No se trata solamente de una especialización, pues suele involucrar principios, normas, características y hasta actores de distinto tipo, como también una falta de coherencia que se va profundizando. Aquí nosotros trataremos dos regímenes del derecho internacional que actúan fuertemente dentro de las relaciones internacionales: el régimen internacional de protección de inversiones y el régimen de protección de los derechos humanos.

A su vez, esta fragmentación del derecho internacional debe abordarse en relación con otros dos fenómenos que pueden verse aparejados, como la inflación y la asimetría normativas.

$\mathrm{El}$ argumento general para justificar la existencia del derecho internacional de las inversiones, mediante tratados bilaterales - conocidos por sus siglas como $\mathrm{TBI}^{2}$ - es que el mismo permite lograr una previsibilidad propicia para fomentar un clima de inversiones para el sector privado. Ello necesariamente dependería, según esta formulación, de la celebración de tratados que protejan estas inversiones extranjeras y de un sistema de solución de controversias que sea favorable a esta protección, para fortalecer de este modo el imperio del derecho (rule of law)

1 Hay once demandas en el CIADI en materia de agua potable y saneamiento: Aguas del Tunari v. Bolivia (ARB/02/3); Biwater Gauff v. Tanzania (ARB/05/22); Compañia Aguas del Aconquija $y$ Vivendi Universal v. Argentina (CIADI, caso ARB/97/3); Aguas Provinciales de Santa Fe, Suez, Sociedad General de Aguas de Barcelona e Interagua Servicios Integrales de Agua v. Argentina (CIADI, caso ARB/03/17); Aguas Cordobesas, Suez, Sociedad General de Aguas de Barcelona v. Argentina (CIADI, caso ARB/03/18); Aguas Argentinas, Suez, Sociedad General de Aguas de Barcelona y Vivendi Universal v. Argentina (CIADI, caso ARB/03/19); Azurix v. Argentina (CIADI, caso ARB/01/12); Azurix (Azurix Mendoza) v. Argentina (CIADI, caso $\mathrm{ARB} / 03 / 30) ;$ SAUR International v. Argentina (CIADI, caso ARB/04/4); Impregilo v. Argentina (CIADI, caso ARB/07/17); Urbaser y Consorcio de Aguas Bilbao Biskaia, Bilbao Biskaia Ur Partzuergoa v. Argentina (CIADI, caso ARB/07/26). Ver: https://icsid.worldbank.org/sp/Pages/ cases/AdvancedSearch.aspx. De este total de demandas en el CIADI sobre el sector de agua potable y saneamiento, puede verificarse que nueve reclamos han sido contra la Argentina, mientras que solo dos fueron contra otros países.

2 La referencia TBI es a los tratados bilaterales de inversión, que también suelen adoptar otras denominaciones como tratados de protección de inversiones, tratados de promoción y protección recíproca de inversiones, tratados de amistad, comercio e inversión, o incluso se encuentran como capítulo de inversiones en los tratados de libre comercio o TLC. 
y dotar de seguridad jurídica y previsibilidad la realización de negocios internacionales.

Sin embargo, este argumento general no se da en los hechos, al menos en casos en los que se esté actuando sobre sectores económicos que tengan vinculación con el derecho internacional de los derechos humanos, como el del agua potable y el saneamiento. Un razonamiento en la lógica inicialmente argumentada indicaría que, para obtener previsibilidad jurídica en materia de agua y saneamiento, resulta necesario celebrar TBI que protejan las inversiones privadas y habiliten jurisdicción a sistemas arbitrales como el del Centro Internacional de Arreglos de Diferencias relativas a Inversiones (CIADI) en al ámbito del Banco Mundial, como mecanismo para la solución de diferencias. Ello ocasiona un caso más de fragmentación del derecho internacional en forma separada de otras áreas, como el derecho internacional de los derechos humanos, con lo cual se obtienen regímenes paralelos que dictan normas sobre agua potable y saneamiento sin que las mismas logren un entrecruzamiento.

Ello provoca un efecto de "enfriamiento regulatorio" que, en algunos casos, puede beneficiar en el corto plazo a las inversiones realizadas en el sector, pero que erosiona los derechos humanos $y$, por ende, causa perjuicios a la sociedad. Con esta erosión de los derechos humanos, la fragmentación del derecho internacional, la multiplicación de normas y la asimetría jurídica entre ciertas normas que se aplican más vigorosamente que otras, no causan una eficacia del derecho, sino todo lo contrario: una proliferación de normas que no respetan un orden jerárquico y producen desequilibrios entre bienes jurídicos protegidos que se ven afectados en un mismo caso pero que son tratados en ámbitos jurídicos distintos, debido a la mencionada fragmentación. Con este desequilibrio y esta ineficacia del derecho para casos tan delicados como el de garantizar el derecho al agua potable y el saneamiento a los habitantes de un territorio determinado, se produce un debilitamiento del rule of law con lo que se evidencia una distancia mayor entre el objetivo pretendido y el resultado obtenido. 
Sin embargo, también hay normas que tratan el agua como un bien social - en términos de un bien común - , por fuera de la apropiación privada individual y más allá de las relaciones sociales mercantiles. Es necesario, entonces, hacer un llamado de atención sobre las contradicciones que pueden desprenderse de la existencia de dos ámbitos jurídicos: un bloque constituido por instrumentos internacionales en vigor y que tratan el agua potable como un bien social, cuyo acceso debe ser garantizado como un derecho humano, y otro bloque formado por un conjunto de normas internacionales y mecanismos jurídico-políticos que consideran el agua como un commodity más, una mercancía liberalizable y regulable por vía del mercado.

\section{LA RELACIÓN ENTRE AGUA, LOS DERECHOS HUMANOS Y LAS INVERSIONES}

El derecho al agua implica agua asequible para todos. Si los servicios de agua son gestionados por terceras partes, el Estado debe prevenirlas sobre los compromisos en torno a un acceso asequible al agua ${ }^{3}$.

Una vinculación convergente del derecho de las inversiones y los derechos humanos es un punto aún no desarrollado dentro del derecho internacional. En los tribunales arbitrales hasta el momento impera una visión restrictiva de esta relación, incluso en comparación con la que los tribunales de inversión poseen respecto del derecho ambiental internacional. Hasta el momento, los tribunales de inversión no han tratado de desarrollar un conjunto coherente de normas relativas a las relaciones entre los derechos humanos y los instrumentos de inversión, y su jurisprudencia al respecto está aún en una etapa muy incipiente ${ }^{4}$.

3 Pierre Thielbörger, The Human Right to Water versus Investors Rights: Double-Dilemma or Pseudo-Conflict?, en Human Rights in International Investment Law and Arbitration, 487-510 (Pierre-Marie Dupuy, Ernst-Ulrich Petersmann \& Francesco Francioni, eds., Oxford University, Oxford, 2009).

4 Moshe Hirsch, Investment Tribunals and Human Rights: Divergent Paths, en Human Rights in International Investment Law and Arbitration, 97-114, (Pierre-Marie Dupuy, Ernst-Ulrich Petersmann \& Francesco Francioni, eds., Oxford University, Oxford, 2009). En este sentido, resalta el laudo del CIADI en el caso Urbaser contra Argentina: a raíz de una reconvención 
Desde 1994, la Constitución Argentina ${ }^{5}$ incorporó tratados de derechos humanos y los llevó a jerarquía constitucional, como el Pacto Internacional de Derechos Económicos, Sociales y Culturales (PIDESC) $)^{6}$, en el cual los organismos de las Naciones Unidas (ONU) han entendido que el acceso al agua y el saneamiento resultan indispensables para la satisfacción de los demás derechos humanos. La misma ONU declaró el derecho humano al agua potable y el saneamiento como básico en 2010. Sin embargo, hay intereses contrapuestos que han quedado evidenciados en casos sometidos a la competencia arbitral internacional en materia de protección de inversiones privadas en el sector de servicios públicos de agua y saneamiento.

Si bien los derechos tienen carácter de relatividad, una visión restrictiva sobre aquellos que resultan esenciales para la vida - como el acceso al agua potable — puede atentar contra la noción de la progresividad de los derechos y de un mayor nivel de bienestar social. En este sentido, y si los derechos en efecto están supeditados a los recursos disponibles, frente a un futuro escenario de crisis en el cual los recursos se muestren cada vez más limitados y escasos, esta visión supone asumir que cada vez deberemos contar con un futuro con menos derechos, menos

de la demandada, se analiza si las empresas concesionarias de la red de distribución de agua potable y saneamiento eran objeto o no de obligaciones en materia de derechos humanos, algo que la empresa negaba absolutamente. El tribunal concluye que son los Estados los sujetos obligados a generar políticas públicas de respeto a los derechos humanos, y en especial al derecho humano al agua, pero que ello no implica que las empresas deban sentirse desobligadas, ya que pesa sobre ellas una obligación de no hacer, es decir, de no interferir con el pleno goce de estos derechos. De este modo, hay responsabilidades en materia de derechos humanos que son atribuibles a los agentes privados y de las cuales no pueden desentenderse, incluso estando por fuera del ámbito contractual o de los TBI. Y sobre ello, el tribunal se definió con jurisdicción para resolver este tipo de cuestiones. Sin embargo, consideró que Argentina no había probado de forma suficiente que la empresa hubiera violado sus obligaciones de no hacer en materia de derechos humanos, y desestimó la reconvención. Centro Internacional de Arreglo de Diferencias relativas a Inversiones, CIADI, Urbaser contra Argentina, caso CIADI ARB/07/26, Laudo, 8 de diciembre de 2016. Disponible en: https://www.italaw.com/cases/1144

5 Argentina, Constitución, Ley 24.430, ordénase la publicación del texto oficial de la Constitución Nacional (sancionada en 1853 con las reformas de los años 1860, 1866, 1898, 1957 y 1994), 15 de diciembre de 1994. Disponible en: http://servicios.infoleg.gob.ar/infolegInternet/ anexos/0-4999/804/norma.htm

6 Organización de Naciones Unidas, ONU, Pacto Internacional de Derechos Económicos, Sociales y Culturales, adoptado y abierto a la firma, ratificación y adhesión por la Asamblea General en su resolución 2200 A (XXI), 16 de diciembre de 1966. Disponible en: http://www.ohchr.org/ SP/ProfessionalInterest/Pages/CESCR.aspx 
libertades y menos bienestar. El desafío es entonces mejorar la administración económica de los recursos para así responder adecuadamente a un mayor bienestar y mayor libertad para la sociedad.

Queda claro que la vinculación entre el régimen de protección de inversiones y el derecho internacional de los derechos humanos existe en puntos claves como los servicios de agua potable y saneamiento ${ }^{7}$. Y esta vinculación deberá quedar reflejada en forma cada vez más presente en los laudos arbitrales, que tendrán que reconocer en forma explícita esta relación.

¿Qué deberes puede imponer el derecho al agua? Frente a esta cuestión, hay que recordar que los deberes de este derecho han sido impuestos al Estado en su deber de regulación ${ }^{8}$. Pero también resulta difícil no caer en una posible contradicción cuando el Estado otorga derechos y se obliga frente a partes que parecen estar en distintos extremos de un conflicto, aun cuando los bienes jurídicos protegidos sean diferentes, como las obligaciones surgidas en materia de protección de la propiedad privada de las inversiones y las obligaciones erga omnes en materia de derechos humanos sobre el acceso al agua potable. El derecho humano al agua no resulta gratuito, sino que va aparejado de una obligación. Pero tal obligación de ejercer el poder de policía por parte del Estado en una mejor y mayor regulación que garantice el derecho al agua va a contrapelo del "enfriamiento regulatorio" que se comprueba como efecto de las demandas ante el CIADI contra los Estados en materia de servicios públicos, y sobre todo en materia de agua y saneamiento. Ello representa un problema serio que debe ser abordado.

7 "La tendencia actual parece indicar que el papel de los derechos humanos en el arbitraje de inversiones seguirá aumentando. Si el sistema de arbitraje es el más adecuado para hacer frente a violaciones de derechos humanos sigue siendo un tema controvertido. La falta de transparencia y de legitimidad son tal vez reproches inevitables y aún está por verse si estos problemas pueden ser resueltos". Entrevista a Mónica Pinto, 27 de junio de 2011. Clara Reiner \& Christoph Schreuer, Human Rights and International Investment Arbitration, en Human Rights in International Investment Law and Arbitration, 82-96 (Pierre-Marie Dupuy, Ernst-Ulrich Petersmann \& Francesco Francioni, eds., Oxford University, Oxford, 2009).

8 Clara Reiner \& Christoph Schreuer, Human Rights and International Investment Arbitration, en Human Rights in International Investment Law and Arbitration, 82-96 (Pierre-Marie Dupuy, Ernst-Ulrich Petersmann \& Francesco Francioni, eds., Oxford University, Oxford, 2009). 
Resultaría sumamente conveniente que pueda entablarse un diálogo entre regímenes jurídicos distintos, como el de la protección de las inversiones y el del derecho internacional de los derechos humanos, para favorecer una mayor coherencia, cooperación, coordinación y sistematización de las obligaciones asumidas por el Estado9. Esta regulación debe ser doble: no solamente en materia de agua y saneamiento, sino también en materia de inversiones, para otorgar un nuevo marco jurídico que dé reglas claras y congruentes a las inversiones extranjeras que deseen actuar en el sector de los servicios públicos. La necesidad de ello radica en que no hay hasta el momento un marco regulatorio en materia de inversiones que fije una pauta al Estado sobre hasta dónde y qué debe proteger en concepto de "derecho de las inversiones": este marco está dado en forma ambigua por los TBI, y esta ambigüedad permite lecturas diversas y muy amplias por parte de los tribunales arbitrales del CIADI, con lo cual realizan interpretaciones que incluso van más allá de la voluntad de los Estados al momento de ratificar estos acuerdos internacionales.

\section{INFLACIÓN Y ASIMETRÍA NORMATIVA EN EL DERECHO INTERNACIONAL}

El fundamento del régimen de inversiones indica que, para obtener la previsibilidad jurídica indispensable para atraer inversiones extranjeras, resulta necesario celebrar TBI que las protejan y prorroguen la jurisdicción local en favor del CIADI. Así se ha generado un régimen autocontenido sin que este conjunto de normas logre un entrecruzamiento con bloques normativos que poseen una prioridad mayor. Ello se suma al enfriamiento regulatorio que en algunos casos pueda beneficiar en el corto plazo a las inversiones realizadas en el sector, pero

9 Ernst-Ulrich Petersmann, Introduction and Summary: 'Administration of Justice' in International Investment Law and Adjudication?, en Human Rights in International Investment Law and Arbitration, 3-44 (Pierre-Marie Dupuy, Ernst-Ulrich Petersmann \& Francesco Francioni, eds., Oxford University, Oxford, 2009). 
que erosiona los derechos humanos y, por ende, causa perjuicios a la ciudadanía en general. Con tal erosión de los derechos humanos, la fragmentación del derecho internacional, la inflación normativa y la asimetría jurídica no se logra una eficacia del derecho sino todo lo contrario: una proliferación de normas que produce desequilibrios entre bienes jurídicos protegidos que se ven afectados para un mismo caso pero que son tratados en ámbitos jurídicos distintos, debido a la fragmentación. Con este desequilibrio y esta ineficacia del derecho para casos tan delicados como garantizar el derecho al agua potable y saneamiento, se produce un debilitamiento del rule of law, con lo que se evidencia una distancia no menor entre el objetivo pretendido y el resultado obtenido.

De proseguir con el actual modo de regulación, nos encontraremos, por un lado, con un tipo de derecho instituido en acuerdos internacionales especializados en materia del agua, congruentes con los derechos humanos, y que consagran el agua como un bien social, pero que no obstante ello, su aplicación no es directamente vinculante (caso de las Resoluciones de la Asamblea General de la ONU) y que bien dependen de la voluntad de los Estados en su ratificación, o se trata también de instrumentos internacionales que pasan a tener un nivel de cumplimiento medianamente laxo (soft law). Del otro lado, vemos una suerte de nova lex mercatoria, con organismos especializados en materia de comercio, que promueven la apertura de mercados y la liberalización de inversiones, con sus propios mecanismos de solución de disputas y sus tribunales arbitrales que garantizan la seguridad jurídica para los actores interesados, pero con una profunda raíz antidemocrática en su proceso de toma de decisiones; sin embargo, no por ello se cuestiona el alto nivel de acatamiento por parte de los Estados involucrados, sobre todo por parte de los países en desarrollo ${ }^{10}$. Esta fuerte disparidad

10 Juan Hernández-Zubizarreta, ¿Lex mercatoria o derechos humanos? Los sistemas de control de las empresas transnacionales, en El negocio de la responsabilidad. Crítica a la responsabilidad social corporativa de las empresas transnacionales, 13-46, 14-15 (Juan Hernández-Zubizarreta $\&$ Pedro Ramiro, eds., Icaria, Barcelona, 2009). 
enseña lo que podemos llamar una asimetría normativa a favor de esa nova lex mercatoria, que muestra también la fuerte evolución de un derecho comercial global frente a un posible - y peligroso - estancamiento del derecho internacional público y de los derechos humanos ${ }^{11}$.

El problema es si cabe la posibilidad de encontrar normas formadas a partir de la voluntad de la comunidad internacional - y no de la voluntad particular de los Estados- que puedan ser "elegibles". En este sentido, ni el ius cogens ni las obligaciones erga omnes pueden ser normas "optativas" para los Estados miembros de la comunidad internacional, pues es, en términos kelsenianos, esta comunidad jurídica la interesada y responsable colectivamente de velar por la plena vigencia de estos derechos ${ }^{12}$. Por lo tanto, al ser los tratados de protección de inversiones parte del derecho internacional público, les resulta aplicable todo el conjunto de obligaciones erga omnes y las normas de derechos humanos.

Si el dilema es entre "derecho al agua" y el "derecho del inversor", el CIADI no ha de ser un foro adecuado para dirimirlo, dado que ofrece tribunales aislados y no un sistema judicial acorde con la estructura jurídica que interprete el derecho internacional general, y tampoco ofrece un tratamiento equilibrado para resolver controversias que involucren derechos humanos fundamentales como el acceso al agua potable y saneamiento, a menos que sea posible aplicar una capacidad procesal pasiva hacia las ETN frente a las cortes regionales de derechos huma-

11 Juan Hernández-Zubizarreta, Las empresas transnacionales frente a los derechos humanos: historia de una asimetría normativa. De la responsabilidad social corporativa a las redes contrahegemónicas transnacionales, 665, 667 (Hegoa, Universidad del País Vasco, Bilbao, 2009). Tal como sostiene el profesor Petersmann: "La dinámica todavía fragmentada del derecho de las inversiones, los derechos humanos, el derecho económico internacional puede llevar a una interpretación más coherente en áreas cada vez más importantes del derecho internacional en la medida en que los jueces y cortes cooperen y en respeto de los derechos humanos y la igualdad soberana de los Estados que deben proteger la legítima diversidad entre ciudadanos y políticas democráticas". Ernst-Ulrich Petersmann, Introduction and Summary: 'Administration of Justice' in International Investment Law and Adjudication?, en Human Rights in International Investment Law and Arbitration, 3-44 (Pierre-Marie Dupuy, Ernst-Ulrich Petersmann \& Francesco Francioni, eds., Oxford University, Oxford, 2009).

12 Hans Kelsen, Derecho y paz en las relaciones internacionales, 125-126 (Fondo de Cultura Económica, FCE, México, 1943). 
nos - algo improbable en el corto plazo-. El laudo de 2016 en el caso Urbaser S.A. contra Argentina (CIADI ARB/07/26) demostró que un tribunal del CIADI puede declararse con jurisdicción para entender sobre una reconvención en la que un Estado puede plantear la responsabilidad internacional de una empresa concesionaria por violaciones al derecho humano al agua. Para ello, el tribunal justificó su competencia basándose en que el TBI entre Argentina y España incluía entre las normas aplicables al caso el derecho internacional general, lo cual le permitió hacer un puente hacia las normas internacionales de derechos humanos. La mayoría de los TBI incluye similares citas al derecho internacional general o al "derecho internacional general referente a la materia”. Sin embargo, el CIADI no es un centro de arbitraje en materia de derechos humanos y desconoce - al menos de una forma vinculante - la jurisprudencia habida en la materia que además resulte aplicable a casos en que estos derechos son violentados por empresas extranjeras inversionistas en el territorio del Estado huésped de esta inversión. Como dijimos, el tema es aún incipiente y está en pleno debate.

En definitiva, la asimetría jurídica y la fragmentación del derecho afectan el rule of law y con ello se afectan a su vez la previsibilidad del sistema jurídico y la seguridad jurídica. De este modo, un sistema imprevisible amenaza tanto los derechos humanos como los derechos de propiedad por igual, quizás no en el corto plazo pero sí más adelante, ya que ello afectará socialmente a los habitantes en términos de tarifas más altas, derechos humanos erosionados, aumentos de impuestos, demandas internacionales contra los Estados, y mayores reclamos sociales.

Los derechos humanos son derechos de carácter objetivo que no pueden, por ello mismo, ser dejados de lado por acuerdos inter partes. El principio de la autonomía de la voluntad de las partes encuentra límites en nociones como el interés público y las normas imperativas del derecho internacional que no pueden ser dejadas de lado por lo pactado entre Estados, mucho menos por lo que el Estado y una empresa acuerden en el contrato de concesión. Aun aquellos autores que todavía no reconocen los 
derechos humanos como ius cogens internacional, sí admiten su aplicación erga omnes, tal como reconoció la Corte Internacional de Justicia, CIJ, en 1970 con el caso Barcelona Traction ${ }^{13}$. En tal sentido, los derechos humanos no son simplemente normas trasplantadas de los ordenamientos internos de los Estados al orden jurídico internacional, sino normas internacionales objetivas que hacen al interés de la comunidad internacional de Estados y que son nacionalmente aplicables por estos para todos ${ }^{14}$. El esclarecimiento judicial de normas sustantivas de tratados internacionales de inversiones en procesos de solución de conflictos económicos transnacionales debe guardar el debido respeto a las obligaciones de los Estados para proteger los derechos humanos y sus obligaciones erga omnes en relación con terceros afectados por disputas de inversiones. Negar la relación de derechos humanos con el régimen de protección de inversiones puede llevar a casos peligrosos en que inversionistas, en connivencia con las autoridades de gobierno anfitrión, eludan las obligaciones de derechos humanos u otras de carácter ius cogens ${ }^{15}$. En ese caso y en medio de la disociación de normas tal como existe hoy, jla violación de obligaciones erga omnes podría justificar la competitividad de una economía? ¿Son los derechos humanos una "variable a ajustar" en pos de una competitividad económica y de la seguridad jurídica para las inversiones?

Creemos que no: ningún Estado puede prescindir de ellos, a diferencia de las condiciones de reciprocidad entre Estados de las que dependen los $\mathrm{TB}^{16}$. Como afirma Mónica Pinto:

13 Cour Internationale de Justice, CIJ, Affaire de la Barcelona Traction, Light and Power Company, Limited, Arrêt du 5 février de 1970. Disponible en: http://www.icj-cij.org/docket/files/50/5387. pdf

14 Mónica Pinto, Las fuentes del derecho internacional en la era de la globalización. Un comentario, en Las fuentes del derecho internacional en la era de la globalización, 13-30, 25-28 (Mónica Pinto, comp., Editorial Universitaria de Buenos Aires, Eudeba, Buenos Aires, 2009).

15 Ernst-Ulrich Petersmann, Introduction and Summary: 'Administration of Justice' in International Investment Law and Adjudication?, en Human Rights in International Investment Law and Arbitration, 3-44 (Pierre-Marie Dupuy, Ernst-Ulrich Petersmann \& Francesco Francioni, eds., Oxford University, Oxford, 2009).

16 Pierre-Marie Dupuy, Unification Rather than Fragmentation of International Law? The Case of International Investment Law and Human Rights Law, en Human Rights in International Investment Law and Arbitration, 45-62 (Pierre-Marie Dupuy, Ernst-Ulrich Petersmann \& Francesco Francioni, eds., Oxford University, Oxford, 2009). 
no se puede decir que a estas alturas de lo que se trata es solamente de un conjunto de aspiraciones morales pateadas hacia adelante, vehiculizadas o motorizadas por un conjunto de ONG o en un ámbito solamente de DDHH. (...) Entonces, creo que en ese punto, tenemos tanto hard law como soft law. La cuestión es saber si yo, negociadora del Estado X con usted, que es un negociador de la empresa $Y$, en materia de agua y saneamiento dentro de una ciudad o de un país, puedo hacer de cuenta que la norma del Pacto no existe... Esto es lo que está viniendo [y] no es lo que hicieron en la década del 90 cuando privatizaron todo... Porque la verdad es que también es muy riesgoso decirle al Estado que se puede sacar la mochila de los derechos humanos solamente cuando tiene buenas posibilidades de hacer caja ${ }^{17}$.

En este mismo sentido, el Estado debe asumir la totalidad de sus obligaciones al momento de proteger las inversiones extranjeras, entre las cuales deberán constar desde ya las obligaciones en materia de derechos humanos. Pero por otro lado, y para que sus expectativas puedan ser consideradas como "legítimas", los inversores también deben tomar debida nota de las obligaciones del Estado derivadas de las obligaciones de derechos humanos ${ }^{18}$.

De persistir esta situación será una oportunidad perdida. El nuevo marco material que surge con la presente crisis mundial abre la posibilidad de revisar el esquema instalado en los años noventa y sintetizar el cambio ideológico que esta crisis está produciendo, para generar un mayor grado de coherencia que asegure el disfrute del derecho humano al agua, la previsión necesaria para dotar de reglas claras al sector privado y un aprovechamiento responsable de los recursos hídricos.

17 Entrevista realizada el 27 de junio de 2011 a Mónica Pinto, decana de la Facultad de Derecho de la Universidad de Buenos Aires, UBA, especialista en derechos humanos y en derecho internacional público.

18 Pierre-Marie Dupuy, Unification Rather than Fragmentation of International Law? The Caso of International Investment Law and Human Rights Law, en Human Rights in International Investment Law and Arbitration, 45-62 (Pierre-Marie Dupuy, Ernst-Ulrich Petersmann \& Francesco Francioni, eds., Oxford University, Oxford, 2009). 


\section{LA FRAGMENTACIÓN DEL DERECHO INTERNACIONAL}

En 2006, el Grupo de Estudio, presidido por el profesor Martti Koskenniemi, presentó un completo informe a la Comisión de Derecho Internacional (CDI), como resultado de su trabajo encomendado tres años antes. Este informe aborda lo que se conoce como la fragmentación del derecho internacional público; el título del informe corresponde al tema agregado por la CDI en 2002 en su plan de trabajo a largo plazo: Fragmentación del derecho internacional: dificultades derivadas de la diversificación y expansión del derecho internacional ${ }^{19}$.

A medida que el derecho internacional fue desarrollándose, progresivamente se evidenció una tendencia hacia la especialización en un número cada vez más creciente de temas, hasta poner en riesgo su homogeneidad como rama jurídica y potenciar su posible fragmentación.

Precisamente, la CDI dedicó parte de sus estudios a este problema creciente del derecho internacional, que se conecta con el tema de este trabajo, pues aborda la posible relación entre dos subramas del derecho internacional - el derecho internacional de las inversiones y el derecho internacional de los derechos humanos - aplicadas a un recurso natural específico: el agua potable. Así, en sus primeras páginas ya evidencia la preocupación creciente de la comunidad internacional y con directa relación con lo que recién comentábamos:

Lo que antes aparecía regido por el "derecho internacional general", se ha convertido en campo de operaciones para sistemas especialistas tales como el "derecho mercantil", el "derecho de los derechos humanos", el "derecho ambiental", el "derecho del mar", el "derecho europeo" e incluso conocimientos tan exóticos y sumamente especializados como el "derecho de las inversiones" o el "derecho internacional de los refugiados", etc. cada uno de los cuales posee sus propios principios e instituciones. El problema, a juicio de los juristas, es que esa legislación y creación de instituciones especializadas tiende a producirse con relativa ignorancia de las actividades

19 Comisión de Derecho Internacional, CDI, Fragmentación del derecho internacional: dificultades derivadas de la diversificación y expansión del derecho internacional (2006). Disponible en: http://legal.un.org/ilc/reports/2006/spanish/chp12.pdf, http://www.refworld.org/cgi-bin/ texis/vtx/rwmain/opendocpdf.pdf?reldoc $=y \&$ docid $=4 \mathrm{a} 716 \mathrm{c} 152$ 
legislativas e institucionales en los campos adyacentes de los principios y prácticas generales del derecho internacional. El resultado son conflictos entre normas o sistemas de normas, prácticas institucionales desviadas y quizá la pérdida de una perspectiva general del derecho ${ }^{20}$.

La tentación de crear regímenes plenamente autónomos cuando es posible hallar normas que puedan apartarse del derecho internacional general a fin de ajustarse a las nuevas necesidades de sectores sociales concretos, es algo real. Entendemos que es preciso allanar posibles contradicciones al evidenciar casos problemáticos que plasmen esta bifurcación entre especialidades y abonen en pos de conservar una coherencia del derecho internacional dentro de la disciplina jurídica. De lo contrario, se estaría avalando un principio de incompatibilidad al favorecer uno u otro régimen según qué perspectiva tome quien esté analizando tal situación: no es posible entender que una obligación internacional puede cumplirse si dejamos de cumplir otra obligación internacional emanada de otra norma jurídica que está a la par de la primera. El derecho internacional debe estar listo para identificar esos casos de incompatibilidad, de modo que puedan crearse esos diálogos entre regímenes fragmentados: se trata en definitiva de un problema de fuentes y de jerarquización normativa. De lo contrario, la erosión del derecho internacional general será una cuestión de tiempo.

El informe de la CDI presenta tres tipos de conflictos entre normas. El tercero de ellos son los conflictos entre dos tipos de normas especiales. Por ejemplo: uno de los argumentos planteados por Argentina ante el Tribunal del CIADI que atendió los casos de Suez contra Argentina (para las concesiones de Aguas Argentinas S.A. y de Aguas Provinciales de Santa Fe S.A.) fue que el actuar del Estado argentino contra las concesiones de las empresas privadas se debía a que resultaba una prioridad para el Estado asegurar el servicio de agua potable y saneamiento en una forma continua y en calidades y cantidades acordes con los

20 Comisión de Derecho Internacional, CDI, Fragmentación del derecho internacional: dificultades derivadas de la diversificación y expansión del derecho internacional, 12 (2006). 
estándares de dignidad a los que Argentina se había obligado internacionalmente en distintos tratados de derechos humanos. El Tribunal no consideró la cuestión de los derechos humanos más que para permitir la presentación de organismos no gubernamentales bajo la figura de amicus curiax. A pesar de ello, en los laudos de fondo, la conexión realizada por el Tribunal entre el caso y su implicancia respecto de cuestiones relativas a los derechos humanos posiblemente afectados tuvo poco alcance.

La propia CDI advirtió que "el principio lex posterior derogat legi priori (la ley posterior deroga la ley anterior) puede ser compensado por jerarquías normativas u opiniones informales sobre la 'pertinencia' o la 'importancia"' 21 , incluso haciendo referencia al artículo 55 del proyecto sobre Responsabilidad internacional del Estado por los hechos internacionalmente ilícitos ${ }^{22}$, en el cual la norma general se aplica supletoriamente a las normas especiales que pudieran regular una situación o tema determinado. Pero también reconoce que "por supuesto, esa facultad no puede ser ilimitada: las normas derogatorias deben tener como mínimo el mismo rango que las derogadas" 23 . Y este es un punto central, ya que las obligaciones que surgen de normas del derecho internacional de los derechos humanos no son jerárquicamente iguales a las obligaciones que surgen del derecho internacional de las inversiones. Obligan colectivamente a la comunidad jurídica internacional y por ello son de carácter erga omnes.

Un incumplimiento por parte del Estado no es excusable frente al riesgo de incumplir con otra obligación: si el Estado decide asumir voluntariamente dos obligaciones, debe cumplir con ambas. Pero hay advertencias en cuanto a una sobrediversificación del derecho internacional por cuanto puede generar una disparidad de normas que provoquen regímenes jurídicos

21 Comisión de Derecho Internacional, CDI, Fragmentación del Derecho Internacional: Dificultades derivadas de la diversificación y expansión del Derecho Internacional, 38 (2006).

22 Ese artículo dice: "Artículo 55. Lex specialis: Los presentes artículos no se aplicarán al caso y en la medida en que las condiciones de existencia de un hecho internacionalmente ilícito, el contenido de la responsabilidad internacional de un Estado o el modo de hacerla efectiva se rijan por normas especiales de derecho internacional".

23 Comisión de Derecho Internacional, CDI, Fragmentación del Derecho Internacional: Dificultades derivadas de la diversificación y expansión del Derecho Internacional, 41 (2006). 
independientes, aislados unos de otros y que, con ello, se provoquen desarrollos normativos en direcciones distintas e incluso contrapuestas. Sobre ello, resulta interesante el planteo realizado por la CDI en cuanto a que parece difícil imaginar cómo pueden los Estados apartarse de la responsabilidad que les cabe del cumplimiento de las obligaciones erga omnes para cumplir las exigencias de determinado self-contained regime.

Asimismo, reviste destacable la cuestión de la jerarquía de normas, que ya esbozamos. A diferencia del derecho interno, el derecho internacional público no está estructurado, en principio, por jerarquías normativas ya que las mismas son producto de una comunidad internacional con una conciencia del carácter de "horizontal" de su sistema jurídico ${ }^{24}$. Simplemente cuenta con el concepto de ius cogens. Tampoco hay orden de prelación entre las fuentes principales de esta rama jurídica, enunciada en el artículo 38 del Estatuto de la Corte Internacional de Justicia ${ }^{25}$. Por ende, tratados, costumbre internacional y principios generales de derecho interno ampliamente reconocidos por las "naciones civilizadas", se hallan en pie de igualdad unas con otras y no hay una graduación valorativa entre ellas ni una preferencia entre las normas jurídicas internacionales. Sin embargo, ello no significa que no haya, de plano, ninguna forma de valoración y jerarquía de normas u obligaciones internacionales. Tal es el caso de las normas de ius cogens y las obligaciones erga omnes del derecho internacional. Esta valoración se halla contemplada en el artículo 103 de la Carta de las Naciones Unidas cuando dice:

24 Comisión de Derecho Internacional, CDI, Fragmentación del derecho internacional: dificultades derivadas de la diversificación y expansión del derecho internacional, 190 (2006).

25 El artículo 38 dice: "Artículo 38. 1. La Corte, cuya función es decidir conforme al derecho internacional las controversias que le sean sometidas, deberá aplicar: a. las convenciones internacionales, sean generales o particulares, que establecen reglas expresamente reconocidas por los Estados litigantes; b. la costumbre internacional como prueba de una práctica generalmente aceptada como derecho; c. los principios generales de derecho reconocidos por las naciones civilizadas; d. las decisiones judiciales y las doctrinas de los publicistas de mayor competencia de las distintas naciones, como medio auxiliar para la determinación de las reglas de derecho, sin perjuicio de lo dispuesto en el artículo 59. 2. La presente disposición no restringe la facultad de la Corte para decidir un litigio ex cequo et bono, si las partes así lo convinieren. Organización de Naciones Unidas, ONU, Estatuto de la Corte Internacional de Justicia. Disponible en: http:// www.icj-cij.org/homepage/sp/icjstatute.php 
Artículo 103. En caso de conflicto entre las obligaciones contraídas por los Miembros de las Naciones Unidas en virtud de la presente Carta y sus obligaciones contraídas en virtud de cualquier otro convenio internacional, prevalecerán las obligaciones impuestas por la presente Carta ${ }^{26}$.

Según la CDI, el artículo 103 no dice que ante un conflicto de normas sea la Carta la que prevalece, sino las obligaciones impuestas por la Carta, lo cual no se refiere a la invalidez de la norma enfrentada con las obligaciones surgidas de la Carta de San Francisco, sino a una razón de establecer prioridades. En este sentido, ambas obligaciones permanecen, pero una de ellas posee una prioridad mayor dada por su jerarquía normativa. Hay, entonces, una categoría normativa sobre la cual no se puede aplicar ningún tipo de derogación, debido a su carácter de fundamental, y en el derecho internacional estas normas son conocidas como el ius cogens internacional al que hacen referencia los artículos 53 y 64 de la Convención de Viena sobre Derecho de los Tratados de $1969^{27}$, como una norma imperativa del derecho internacional general.

Una distinción diferente a las obligaciones de la Carta del artículo 103 y a las normas ius cogens, son las obligaciones erga omnes. Si bien estas obligaciones no constituyen claramente una "jerarquía superior" - al estilo del ius cogens, por ejemplo-, estas obligaciones designan ámbitos de aplicación del derecho pues obligan a la comunidad internacional en su conjunto, es decir, a todos los Estados más allá de su adhesión formal y expresa a la obligación en términos positivos mediante la ratificación de un tratado, por ejemplo, así como también pueden ser invocadas por cualquier Estado, en cuanto a la responsabilidad que pueda caberle al Estado infractor de este tipo de obligaciones. Y si bien el valor de este tipo de obligaciones todavía es un debate inconcluso de la doctrina internacional, la idea de la aplicación

26 Organización de Naciones Unidas, ONU, Carta de las Naciones Unidas, San Francisco, 26 de junio de 1945. Disponible en: http://www.un.org/es/charter-united-nations/index.html

27 Organización de Naciones Unidas, ONU, Convención de Viena sobre Derecho de los Tratados, Viena, 23 de mayo de 1969. Disponible en: http://www.oas.org/xxxivga/spanish/reference_docs/ convencion_viena.pdf 
de ciertas obligaciones que resultan ser oponibles a todos es una práctica arraigada dentro del derecho internacional ${ }^{28}$, por cuanto no puede negarse su existencia. Fue el caso Barcelona Traction el que por primera vez en el derecho internacional adoptó la denominación de erga omnes a ciertas obligaciones de los Estados que van más allá de obligaciones recíprocas entre Estados, sino que constituyen obligaciones que se asumen frente a toda la comunidad de Estados y que por su carácter fundamental y la importancia de los derechos involucrados, a todos los Estados les incumbe velar por los intereses jurídicos protegidos por tales obligaciones ${ }^{29}$. Se trata de obligaciones relativas a normas secundarias, no a normas primarias.

La mayoría de las obligaciones erga omnes proviene del campo del derecho internacional de los derechos humanos y del derecho internacional humanitario, ámbitos en los cuales los Estados no generan obligaciones en forma bilateral sino que van dirigidas a toda la comunidad de Estados en general y a todos los ciudadanos de la comunidad internacional. En este tipo de obligaciones, el Estado asume una responsabilidad de respetar los derechos humanos independientemente de cómo se estén comportando otros Estados. Allí radica la importancia de las obligaciones erga omnes: poseen una exigibilidad de carácter general — es decir, a toda la generalidad de Estados-, con base en la presunción de que el acto que pudiera violarlas atenta contra ciertos valores o intereses "de todos" o, en la terminología del asunto Barcelona Traction, de la "comunidad internacional en su conjunto", es decir, aquellas obligaciones en cuyo cumplimiento cada Estado tiene un interés jurídico ${ }^{30}$ y que, por lo tanto, resultan exigibles en todo momento sin que pueda haber momentos en que estas obligaciones puedan suspenderse o quedar eximidas, pues el

28 Comisión de Derecho Internacional, CDI, Fragmentación del derecho internacional: dificultades derivadas de la diversificación y expansión del derecho internacional, 221-222 (2006).

29 Comisión de Derecho Internacional, CDI, Fragmentación del derecho internacional: dificultades derivadas de la diversificación y expansión del derecho internacional, 225 (2006).

30 Comisión de Derecho Internacional, CDI, Fragmentación del derecho internacional: dificultades derivadas de la diversificación y expansión del derecho internacional, 228, 234-235 (2006). 
Estado se obliga a cumplirlas en todo momento, sin que se admitan normas de suspensión de estos derechos ${ }^{31}$.

Ello no significa que las obligaciones erga omnes (multilaterales, no recíprocas, basadas en valores comunes y por ende exigibles sin excepciones) sean a su vez normas ius cogens (imperativas e inderogables a menos que sea por otras posteriores y del mismo carácter). Mientras estas últimas se centran en su carácter de inderogabilidad y oponibilidad frente a cualquier otra norma, las primeras se centran no en su validez, sino en el interés jurídico protegido que además concierne a toda la comunidad internacional. En este sentido, pocas dudas pueden caber respecto a que las obligaciones erga omnes puedan ser equiparadas al resto de las obligaciones que sí son contraídas con base en una reciprocidad, como pueden ser el compromiso mutuo por vía convencional de la protección de las inversiones extranjeras en un Estado, pues protegen un bien jurídico esencial del derecho internacional público: el respeto a los derechos humanos ${ }^{32}$. Está claro que la protección de inversiones no necesariamente afecta obligaciones erga omnes, pero sí hay ciertas áreas que, por el tipo de obligaciones que acarrean o de sectores que involucran, se vinculan directamente o que - en palabras de los laudos del CIADI de Aguas Argentinas y Aguas Provinciales de Santa Fe sobre el amicus curic presentado- - "podrían plantear una amplia gama de cuestiones complejas en materia de derecho público e internacional, incluidas consideraciones relativas a derechos humanos" ${ }^{\prime 33}$.

31 Entrevista a Mónica Pinto, 27 de junio de 2011.

32 La CDI incluso ha comparado al bloque de normas ius cogens y de obligaciones erga omnes del derecho internacional con las normas de orden público de los derechos internos de los Estados, aplicando en forma análoga las categorías de las primeras con estas últimas. Comisión de Derecho Internacional, CDI, Fragmentación del derecho internacional: dificultades derivadas de la diversificación y expansión del derecho internacional, 230 (2006).

33 Centro Internacional de Arreglo de Diferencias relativas a Inversiones, CIADI, Aguas Argentinas, S.A., Suez, Sociedad General de Aguas de Barcelona, S.A. y Vivendi Universal, S.A. contra República Argentina, caso CIADI ARB/03/19, Resolución en respuesta a la petición de participación como amicus curice, 19 de mayo de 2005, 9-10, párr. 19. Disponible en: https:// www.italaw.com/cases/1057 Centro Internacional de Arreglo de Diferencias relativas a Inversiones, CIADI, Azurix Corp. contra República Argentina, Laudo de fondo, caso CIADI ARB/01/12, 14 de julio de 2006, 8-9, párr. 18. Disponible en: http://www.iisd.org/pdf/2006/ itn_azurix_argentina_july.pdf, https://www.italaw.com/cases/118 
La fragmentación es, para la CDI, un aspecto de la mundialización, con la que se generan redes de cooperación especializadas técnicamente y que poseen un alcance global, pero que también son difíciles de regular por medio del derecho internacional tradicional ${ }^{34}$, motivo por el cual se necesitan ámbitos jurídicos especializados aun cuando ello fragmente el derecho internacional.

\section{UN DESARROLLO PROGRESIVO DE MÚLTIPLES VELOCIDADES}

A la fragmentación del derecho internacional y la inflación normativa se suma el problema de encontrar ciertos grados de validez coercitiva de normas que no siempre se condicen con el rango de jerarquías habido en el sistema jurídico, lo que genera un desequilibrio entre las normas existentes dentro del derecho internacional ${ }^{35}$.

El desarrollo de subsistemas en forma tan disociada uno del otro como han sido el derecho internacional de los derechos humanos y el derecho internacional de las inversiones, también ha provocado grados de especialización y profundización de cada uno de ellos de modo que cada cual ha podido generar sus propios sistemas de control, aplicación y sanción. De esta manera, el desarrollo progresivo del derecho internacional se ha dado al mismo tiempo que su fragmentación. Incluso se observa que el estándar de protección proveído por los instrumentos jurídicos de derechos humanos es más bajo que el contenido en los tratados de inversiones y en los contratos. Por ende, el desarrollo de áreas de experticia como el derecho internacional de las inversiones no ha sido uniforme respecto de otras como el derecho internacional de los derechos humanos, además de haberse dado sin un diálogo entre ambas.

34 Comisión de Derecho Internacional, CDI, Fragmentación del derecho internacional: dificultades derivadas de la diversificación y expansión del derecho internacional, 228, 281 (2006).

35 Juan Hernández-Zubizarreta, Un nuevo derecho corporativo transnacional. El carácter feudal de los ordenamientos jurídicos globales, en Gobernanza y manejo sustentable del agua Governance and Sustainable Management of Water, 195-220, 197 (Griselda D. Capaldo, ed., Mnemosyne, Buenos Aires, 2011). 
Hasta el momento, ha habido un desarrollo progresivo para$l e l o$, en el cual el derecho internacional de las inversiones (que muchas veces también está aparejado con el derecho relativo al comercio internacional) ha crecido más rápido y de forma más profunda que el derecho internacional de los derechos humanos, $\mathrm{y}$ en una forma completamente disociada entre ambos.

Por un lado, podemos encontrar una sumatoria de tratados bilaterales de protección de inversiones y otros que son tratados de libre comercio que incorporan capítulos de inversiones que son asimilables - cuando no idénticos-a los TBI. Este conjunto de tratados actúa como red de relaciones jurídicas bilaterales en un marco de cierta multilateralidad otorgada por cláusulas como la de la nación más favorecida (CNMF). Por el otro lado, encontramos el derecho internacional de los derechos humanos, de carácter multilateral y con obligaciones erga omnes que importan a toda la comunidad internacional y no solamente a los Estados parte de las convenciones que lo forman.

Esta especie de disociación entre el derecho internacional de las inversiones y el derecho internacional de los derechos humanos encuentra en el caso del agua un punto común, para el que resulta necesario establecer el entrecruzamiento que los vincule para evitar consecuencias que pudieran ser perjudiciales tanto para asegurar un clima estable para las inversiones como también la plena vigencia de los derechos humanos en el orden nacional e internacional, algo poco estudiado en el derecho internacional ${ }^{36}$.

El derecho de las inversiones que, aun siendo formalmente bilateral, ha formado un complejo sistema de vínculos jurídicos tanto en las relaciones entre normas (mediante la CNMF), como también dentro del sistema de solución de controversias que ha creado (los tribunales del CIADI), pues aun siendo ad hoc acostumbran citar jurisprudencia de otros tribunales arbitrales

36 Juan Pablo Bohoslavsky, Tratados de protección de las inversiones e implicaciones para la formulación de políticas públicas (especial referencia a los servicios de agua potable y saneamiento), 17 (Comisión Económica para América Latina y el Caribe, CEPAL, Santiago de Chile, 2010). Disponible en: http://repositorio.cepal.org/bitstream/handle/11362/3769/1/ S2010545_es.pdf 
y aplicar analogía a sus casos quizás debido a la profunda raíz del common law, de la cual viene la mayoría de sus árbitros. El resultado de este desarrollo ha sido un sistema de protección de inversiones de carácter vinculante, coercitivo y eficaz que encuentra en los laudos de los tribunales del CIADI resoluciones inapelables que los Estados partes - generalmente condenados — tienen la obligación de acatar.

Paralelamente está el derecho internacional de los derechos humanos que, aun siendo anterior a los mecanismos de protección de inversiones, avanza a un paso mucho más lento y menos coercitivo. Sus normas todavía tienen un peso relativo en su aplicación coercitiva: muchas de ellas son un soft law que, aunque siendo derecho positivo, no resulta vinculante para los Estados. En el centro de este bloque normativo está lo que puede ser un "hard law mínimo" y que se halla en instrumentos jurídicos como el Pacto de Derechos Económicos, Sociales y Culturales o la Declaración Universal de los Derechos Humanos, que en el caso argentino tienen jerarquía constitucional en ambos casos.

Pero las normas de este fragmento del derecho internacional, a pesar de tener un perfil más importante que el de las normas sobre inversiones, resultan de una aplicación más difícil. En este sentido, las voces que más duramente critican esta relación asimétrica entre ambos subsistemas de normas observan "una estructura formal de norma jurídica pero con vocación de construir ámbitos de derecho blando, que permiten a las multinacionales funcionar con plenas garantías económicas y con criterios unilaterales entroncados en el Derecho Internacional de Comercio y al margen de la función tuitiva del Derecho Internacional de los Derechos humanos"37.

Estos fuertes desequilibrios que se producen entre el mercado y los derechos humanos son muestra de una asimetría normativa entre el derecho internacional de protección de las inversiones y el derecho internacional de los derechos humanos que, lejos

37 Juan Hernández-Zubizarreta, Las empresas transnacionales frente a los derechos humanos: historia de una asimetría normativa. De la responsabilidad social corporativa a las redes contrahegemónicas transnacionales, 664 (Hegoa, Universidad del País Vasco, Bilbao, 2009). 
de otorgar al sistema jurídico una mayor seguridad, aporta incoherencia puesto que socava la validez de obligaciones de importancia mayor (las obligaciones erga omnes de los derechos humanos $)^{38}$ en pos de favorecer aquellas emanadas de otro selfcontained regime pero que no goza del rango de las primeras. Nos hallamos ante una asimetría normativa producto de un desarrollo progresivo de regímenes autónomos que han crecido a velocidades diferentes ${ }^{39}$, y han pasado por alto que el derecho internacional de los derechos humanos es de carácter público frente al derecho de las inversiones, de índole privada ${ }^{40}$, como también el carácter objetivo de los derechos humanos frente a la "subjetividad" de las obligaciones surgidas a partir de tratados bilaterales como los de protección de inversiones que dependen de esta bilateralidad recíproca entre Estados soberanos y no del carácter objetivo que le otorga todo el sistema, como ocurre con las obligaciones erga omnes ${ }^{41}$.

Está claro que este entrecruzamiento en el caso del servicio de agua potable no deslinda las responsabilidades del Estado y tampoco la protección de las inversiones garantizadas por los TBI. Pero ya ha habido distintas advertencias, tanto en algunos laudos del CIADI como sobre todo en las defensas planteadas por los Estados de que los incumplimientos contractuales por parte de las empresas han sido motivos fuertes por los cuales se han quitado las concesiones en un plazo mucho menor a los acordados (30 o 40 años), lo cual configuró, para los tribunales del CIADI, en la mayoría de los casos de responsabilidad internacional la violación del TBI.

38 Comisión de Derecho Internacional, CDI, Fragmentación del derecho internacional: dificultades derivadas de la diversificación y expansión del derecho internacional, 230, 236, 41, 228, 281 (2006).

39 Aniza García, El derecho humano al agua, 113, 168-169 (Trotta, Madrid, 2008).

40 Moshe Hirsch, Investment Tribunals and Human Rights: Divergent Paths, en Human Rights in International Investment Law and Arbitration, 97-114 (Pierre-Marie Dupuy, Ernst-Ulrich Petersmann \& Francesco Francioni, eds., Oxford University, Nueva York, 2009).

41 Pierre-Marie Dupuy, Unification Rather than Fragmentation of International Law? The Case of International Investment Law and Human Rights Law, en Human Rights in International Investment Law and Arbitration, 45-62 (Pierre-Marie Dupuy, Ernst-Ulrich Petersmann \& Francesco Francioni, eds., Oxford University, Oxford, 2009). 
Pero "el tema de que exista un sistema de solución de controversias del lado de las inversiones que parezca mucho más eficaz y que sea mucho más frecuente, no inhibe la capacidad del Estado de tener argumentos para poder incidir en la regulación de un producto"42. Nos encontramos entonces frente a una situación jurídica problemática. Esta situación provoca un estado de "enfriamiento regulatorio" que coloca al Estado en una disyuntiva sin salida aparente:

Si bien los laudos arbitrales no son homogéneos - lo cual se agrava por el hecho de que no existe un mecanismo institucional de unificación de la jurisprudencia arbitral (Van Harten, 2006)—, si se puede observar una tendencia general a aceptar interpretaciones expansivas de los derechos de los inversores, y en materia de expropiaciones esto se puede visualizar notoriamente. Una de las mayores preocupaciones que genera tal tendencia es que esa mayor rigurosidad en el juzgamiento de la expropiación indirecta puede producir como efecto el llamado enfriamiento regulatorio (Waincymer, 2009) que es la inhibición que sufren las respectivas agencias estatales para modificar y mejorar la regulación, pues temen de alguna manera a las consecuencias legales que puedan sobrevenir (Rose-Ackerman \& Rossi, 2000 ${ }^{43}$.

De esta forma se acaba asegurando el derecho internacional de las inversiones en desmedro de los derechos de los usuarios a una mejor y mayor regulación, como también la progresividad de un mayor y mejor alcance de los derechos humanos en cuanto al acceso al agua potable para aquellos que no lo tienen, a un nivel de vida adecuado, a una mejor salud, alimentación, vivienda, etc.

Creemos que se está llegando a un punto en que el riesgo de continuar con esta asimetría jurídica puede llevar a resultados muy alejados de la seguridad y previsibilidad que se pretende del derecho como disciplina científica. El resultado podría ser una ruptura de ciertos regímenes autónomos respecto del derecho internacional general, con sistemas de resolución de conflictos propios, normas propias, reglas propias, y un lenguaje propio y

42 Entrevista a Mónica Pinto, 27 de junio de 2011.

43 Juan Pablo Bohoslavsky, Tratados de protección de las inversiones e implicaciones para la formulación de políticas públicas (especial referencia a los servicios de agua potable y saneamiento), 37 (Comisión Económica para América Latina y el Caribe, CEPAL, Santiago de Chile, 2010). 
autorreferencial, para alejarse de toda relación con el derecho internacional genérico o con otros regímenes que imponen obligaciones de mayor jerarquía dentro del derecho internacional. 
ConClusiones

De proseguir con el actual modo de regulación, nos encontraremos, por un lado, con un tipo de derecho instituido en acuerdos internacionales especializados en materia de agua, congruentes con los derechos humanos y que consagran el agua como un bien social, pero que no obstante ello su aplicación no es directamente vinculante (Resoluciones de la Asamblea General de la ONU) y que bien dependen de la voluntad de los Estados en su ratificación, o se trata también de instrumentos internacionales que pasan a tener un nivel de cumplimiento medianamente laxo (soft law). Del lado opuesto, nos encontraremos con un conjunto de normas internacionales que conforman un bloque jurídico mercantil, una lex mercatoria, con organismos especializados en materia de comercio, que promueven la apertura de mercados, liberalización de inversiones, con sus propios mecanismos de solución de disputas y sus tribunales arbitrales que garantizan la seguridad jurídica para los actores interesados, pero con una profunda raíz antidemocrática en su proceso de toma de decisiones, y que no por ello cuestiona el alto nivel de acatamiento por parte de los Estados involucrados, sobre todo por parte de los países en desarrollo ${ }^{44}$.

Esta fuerte disparidad enseña lo que podemos llamar una asimetría normativa a favor de la lex mercatoria, que muestra también la fuerte evolución de un derecho comercial global frente a un posible - y peligroso - estancamiento del derecho internacional público y de los derechos humanos ${ }^{45}$.

Tal como sostiene el profesor Ernst-Ulrich Petersmann:

44 Juan Hernández-Zubizarreta, ¿Lex mercatoria o derechos humanos? Los sistemas de control de las empresas transnacionales, en El negocio de la responsabilidad. Crítica a la responsabilidad social corporativa de las empresas transnacionales, 13-46, 14-15 (Juan Hernández-Zubizarreta \& Pedro Ramiro, eds., Icaria, Barcelona, 2009).

45 Juan Hernández-Zubizarreta, Las empresas transnacionales frente a los derechos humanos: historia de una asimetría normativa. De la responsabilidad social corporativa a las redes contrahegemónicas transnacionales, 665, 667 (Hegoa, Universidad del País Vasco, Bilbao, 2009). 
La dinámica todavía fragmentada del derecho de las inversiones, los derechos humanos, el derecho económico internacional puede llevar a una interpretación más coherente en áreas cada vez más importantes del derecho internacional en la medida en que los jueces y cortes cooperen y en respeto de los derechos humanos y la igualdad soberana de los Estados que deben proteger la legítima diversidad entre ciudadanos y politicas democráticas ${ }^{46}$.

Para Petersmann, puede haber una tendencia a disminuir la fragmentación gracias a los laudos y sentencias de cortes siempre que usen principios comunes de procedimiento pero que también promuevan una coherencia mutua, una interpretación de estándares de protección legal acordados internacionalmente sobre regulación, expropiación y sobre los mecanismos de solución de disputas. Él entiende que cada vez más tribunales arbitrales demandan que las reglas en inversiones deberían constituirse en conformidad con las obligaciones de cada Estado dentro de organismos como la Organización Mundial del Comercio, OMC. Ello quizás implique la búsqueda de una posible resignificación de este organismo que ayude a sacar del cono de sombra en que están sus negociaciones - la Ronda de Doha-, iniciadas en 2001 y convertidas en la ronda de negociaciones más larga de la que se tenga memoria. Pero este punto no es materia de este trabajo.

Hans Kelsen definía ya en la década de 1940 que dentro del derecho internacional la mayoría de las obligaciones se establece por medio del consentimiento de cada Estado en adherir o no a esta obligación. Es lo que conocemos como derecho internacional particular en oposición al derecho internacional general o común ${ }^{47}$. Exceptuando que estemos, por ejemplo, frente a una costumbre internacional - en la que incluso el Estado podrá haber expresado su disconformidad en forma repetida y sistemática (figura del objetor persistente) para que esta costumbre interna-

46 Ernst-Ulrich Petersmann, Introduction and Summary: 'Administration of Justice' in International Investment Law and Adjudication?, en Human Rights in International Investment Law and Arbitration, 3-44 (Pierre-Marie Dupuy, Ernst-Ulrich Petersmann \& Francesco Francioni, eds., Oxford University, Oxford, 2009).

47 Hans Kelsen, Derecho y paz en las relaciones internacionales, 53 (Fondo de Cultura Económica, FCE, México, 1943). 
cional en formación no le resulte oponible - , las obligaciones internacionales surgen con base en una relación de reciprocidad en la cual dos o más Estados deciden obligarse mutuamente. De este modo, la mayor parte de las obligaciones internacionales surgen a partir de un principio de descentralización estático ${ }^{48}$, por el cual son relativamente pocas las obligaciones dentro del derecho internacional creado por la propia comunidad y no a partir del compromiso de los Estados en forma individual. El problema es si cabe la posibilidad de encontrar normas formadas a partir de la voluntad de la comunidad internacional $-\mathrm{y}$ no de la voluntad particular de los Estados - que puedan ser "elegibles". En este sentido, tanto el ius cogens como las obligaciones erga omnes no pueden ser normas que resultan "optativas" para los Estados miembros de la comunidad internacional, pues es, en términos kelsenianos, esta comunidad jurídica la interesada y responsable colectivamente de velar por la plena vigencia de estos derechos ${ }^{49}$. Por lo tanto, al ser los tratados de protección de inversiones parte del derecho internacional público, les resulta aplicable todo el conjunto de obligaciones erga omnes como las normas de derechos humanos.

Tal vez estamos arribando a un punto en el que es preciso definir entre una estructura fragmentada ad hoc y TBI bilaterales o si suscribimos definitivamente a este "sistema" de inversiones dentro del derecho internacional y en conformidad con el ius cogens y las obligaciones erga omnes existentes. No se trata de "optar" por una estructura jurídica internacional fragmentada o centralizada, pues no es posible una "opción": más allá de las fuerzas por centralizar esta rama del derecho, la fragmentación y la descentralización son ya características intrínsecas del derecho internacional público. El problema que se presenta es conjugar un subsistema como el régimen internacional de las inversiones con obligaciones erga omnes como los derechos humanos. Y ello resulta un desafío en tanto y en cuanto se han desarrollado

48 Hans Kelsen, La paz por medio del derecho, 48-54, $75-78$ (Losada, Buenos Aires, 1946).

49 Hans Kelsen, Derecho y paz en las relaciones internacionales, 125-126 (Fondo de Cultura Económica, FCE, México, 1943). 
regímenes jurídicos diferentes, con distintos alcances y múltiples velocidades en su historia, por lo cual no resulta extraño que algunas de las resoluciones emanadas de estos regímenes aparezcan como contradictorias ${ }^{50}$.

Tal cuestión plantea el dilema de comenzar a ordenar el derecho internacional desde algunos casos puntuales en que se detecten entrecruzamientos problemáticos y evitar así que, por su gran fragmentación, algunos subsistemas acaben desarrollando mayores y más efectivas obligaciones que aquellos subsistemas que resultan prioritarios - como los derechos humanos - por el tipo de obligaciones que involucran. El objetivo es evitar desequilibrios entre los distintos self-contained regimes y la asimetría normativa que ello implica, a fin de focalizar un desarrollo progresivo equilibrado del derecho internacional, entre las normas de protección de inversiones y los derechos humanos.

Por ende, es posible un aumento de los reclamos por parte de la sociedad civil contra el Estado como blanco de demandas para solucionar el déficit en la eficacia del derecho ambiental o en el suministro de servicios públicos esenciales, y ello incumbe a los distintos poderes del Estado -Ejecutivo, Legislativo y Judicial ${ }^{51}$. Pero también se torna previsible un incremento de reclamos hacia organismos como el CIADI y el régimen internacional de inversiones en la medida en que afecten los derechos sociales, generen una asimetría normativa y atenten contra la previsibilidad y la seguridad jurídica en el largo plazo, lo que vuelve aquellas promesas del sistema en su propia contra como deudas incumplidas.

50 Pierre Thielbörger, The Human Right to Water versus Investors Rights: Double-Dilemma or Pseudo-Conflict?, en Human Rights in International Investment Law and Arbitration, 487-510 (Pierre-Marie Dupuy, Ernst-Ulrich Petersmann \& Francesco Francioni, eds., Oxford University, Oxford, 2009).

51 Jona Razzaque, Public Participation in Water Governance, en The Market Alternative, en The Evolution of the Law and Politics of Water, 353-372, 355 (Joseph W. Dellapenna \& Joyeeta Gupta, eds., Springer, Villanova, Amsterdam, 2009). Jaume Delclòs i Ayats, La gestión pública con participación y control social: hacia el derecho humano al agua, en Agua, un derecho y no una mercancía, 137-152, 144-150 (Jaume Delclòs, coord., Icaria, Barcelona, 2009). 


\section{BIBLIOGRAFÍA}

\section{Libros}

Dupuy, Pierre-Marie; Petersmann, Ernst-Ulrich \& Francioni, Francesco, Human Rights in International Investment Law and Arbitration (Oxford University, Oxford, 2009).

García, Aniza, El derecho humano al agua (Trotta, Madrid, 2008).

Hernández-Zubizarreta, Juan, Las empresas transnacionales frente a los derechos humanos: historia de una asimetría normativa. De la responsabilidad social corporativa a las redes contrahegemónicas transnacionales (Hegoa, Universidad del País Vasco, Bilbao, 2009).

Kelsen, Hans, Derecho y paz en las relaciones internacionales (Fondo de Cultura Económica, FCE, México, 1943).

Kelsen, Hans, La paz por medio del derecho (Losada, Buenos Aires, 1946).

\section{Colaboración en obras colectivas}

Delclòs i Ayats, Jaume, La gestión pública con participación y control social: hacia el derecho humano al agua, en Agua, un derecho y no una mercancia, 137-152 (Jaume Delclòs y Ayats, coord., Icaria, Barcelona, 2009).

Dupuy, Pierre-Marie, Unification Rather than Fragmentation of International Law? The Case of International Investment Law and Human Rights Law, en Human Rights in International Investment Law and Arbitration, 45-62 (Pierre-Marie Dupuy, ErnstUlrich Petersmann \& Francesco Francioni, eds., Oxford University, Oxford, 2009).

Hernández-Zubizarreta, Juan, ¿Lex mercatoria o derechos humanos? Los sistemas de control de las empresas transnacionales, en El negocio de la responsabilidad. Crítica a la responsabilidad social corporativa de las empresas transnacionales, 13-46 (Juan Hernández-Zubizarreta \& Pedro Ramiro, eds., Icaria, Barcelona, 2009).

Hernández-Zubizarreta, Juan, Un nuevo derecho corporativo transnacional. El carácter feudal de los ordenamientos juridicos globales, en Gobernanza y manejo sustentable del agua - Governance and Sustainable Management of Water, 195-220 (Griselda D. Capaldo, ed., Mnemosyne, Buenos Aires, 2011).

Hirsch, Moshe, Investment Tribunals and Human Rights: Divergent Paths, en Human Rights in International Investment Law and Arbitration, 97-114 (Pierre-Marie Dupuy, Ernst-Ulrich Petersmann \& Francesco Francioni, eds., Oxford University, Oxford, 2009).

Petersmann, Ernst-Ulrich, Introduction and Summary: 'Administration of Justice' in International Investment Law and Adjudication?, en Human Rights in International Investment Law and Arbitration, 3-44 (Pierre-Marie Dupuy, Ernst-Ulrich Petersmann \& Francesco Francioni, eds., Oxford University, Oxford, 2009).

Pinto, Mónica, Las fuentes del derecho internacional en la era de la globalización. Un comentario, en Las fuentes del derecho internacional en la era de la globalización, 13-30 (Mónica Pinto, comp., Editorial Universitaria de Buenos Aires, Eudeba, 
Buenos Aires, 2009).

Razzaque, Jona, Public Participation in Water Governance, en The Market Alternative, en The Evolution of the Law and Politics of Water, 353-372 (Joseph W. Dellapenna \& Joyeeta Gupta, eds., Springer, Villanova, Amsterdam, 2009).

Reiner, Clara \& Schreuer, Christoph, Human Rights and International Investment Arbitration, en Human Rights in International Investment Law and Arbitration, 82-96 (Pierre-Marie Dupuy, Ernst-Ulrich Petersmann \& Francesco Francioni, eds., Oxford University, Oxford, 2009).

Thielbörger, Pierre, The Human Right to Water versus Investors Rights: Double-Dilemma or Pseudo-Conflict?, en Human Rights in International Investment Law and Arbitration, 487-510 (Pierre-Marie Dupuy, Ernst-Ulrich Petersmann \& Francesco Francioni, eds., Oxford University, Oxford, 2009).

\section{Working paper}

Bohoslavsky, Juan Pablo, Tratados de protección de las inversiones e implicaciones para la formulación de políticas públicas (especial referencia a los servicios de agua potable y saneamiento) (Comisión Económica para América Latina y el Caribe, CEPAL, Santiago de Chile, 2010). Disponible en: http://repositorio.cepal. org/bitstream/handle/11362/3769/1/S2010545_es.pdf

\section{Tratados internacionales y normatividad internacional}

Organización de Naciones Unidas, ONU, Carta de las Naciones Unidas, San Francisco, 26 de junio de 1945. Disponible en: http://www.un.org/es/charter-united-nations/ index.html

Organización de Naciones Unidas, ONU, Convención de Viena sobre Derecho de los Tratados, Viena, 23 de mayo de 1969. Disponible en: http://www.oas.org/xxxivga/ spanish/reference_docs/convencion_viena.pdf

Organización de Naciones Unidas, ONU, Estatuto de la Corte Internacional de Justicia. Disponible en: http://www.icj-cij.org/homepage/sp/icjstatute.php

Organización de Naciones Unidas, ONU, Pacto Internacional de Derechos Económicos, Sociales y Culturales, adoptado y abierto a la firma, ratificación y adhesión por la Asamblea General en su resolución 2200 A (XXI), 16 de diciembre de 1966. Disponible en: http://www.ohchr.org/SP/ProfessionalInterest/Pages/CESCR.aspx

\section{Normatividad argentina}

Argentina, Constitución, Ley 24.430, ordénase la publicación del texto oficial de la Constitución Nacional (sancionada en 1853 con las reformas de los años 1860, 1866, 1898, 1957 y 1994), 15 de diciembre de 1994. Disponible en: http://servicios. infoleg.gob.ar/infolegInternet/anexos/0-4999/804/norma.htm 


\section{Jurisprudencia internacional}

Cour Internationale de Justice, CIJ, Affaire de la Barcelona Traction, Light and Power Company, Limited, Arrêt du 5 février de 1970. Disponible en: http://www.icj-cij. org/docket/files/50/5387.pdf

\section{Jurisprudencia CIADI}

Centro Internacional de Arreglo de Diferencias relativas a Inversiones, CIADI, Aguas Argentinas, S.A., Suez, Sociedad General de Aguas de Barcelona, S.A. y Vivendi Universal, S.A. contra República Argentina, caso CIADI ARB/03/19, Resolución en respuesta a la petición de participación como amicus curice, 19 de mayo de 2005. Disponible en: https://www.italaw.com/cases/1057

Centro Internacional de Arreglo de Diferencias relativas a Inversiones, CIADI, Azurix Corp. contra República Argentina, Laudo de fondo, caso CIADI ARB/01/12, 14 de julio de 2006. Disponible en: http://www.iisd.org/pdf/2006/itn_azurix_argentina_july.pdf, https://www.italaw.com/cases/118

Centro Internacional de Arreglo de Diferencias relativas a Inversiones, CIADI, $U r$ baser contra Argentina, caso CIADI ARB/07/26, Laudo, 8 de diciembre de 2016. Disponible en: https://www.italaw.com/cases/1144

\section{Informes, reportes, documentos}

Comisión de Derecho Internacional, CDI, Fragmentación del derecho internacional: dificultades derivadas de la diversificación y expansión del derecho internacional (2006). Disponible en: http://legal.un.org/ilc/reports/2006/spanish/ chp12.pdf, http://www.refworld.org/cgi-bin/texis/vtx/rwmain/opendocpdf. pdf?reldoc $=$ y\&docid $=4 \mathrm{a} 716 \mathrm{c} 152$

\section{Direcciones web}

https://icsid.worldbank.org/sp/Pages/cases/AdvancedSearch.aspx 